\title{
Two-sided Matching Decision under Uncertain Score Environment
}

\author{
Qi Yue \\ School of Information Management, Jiangxi University of Finance and Economics, \\ Nanchang, China \\ yueqichina@126.com
}

\begin{abstract}
In order to solve the two-sided matching problem based on uncertain score information, $a$ new method is presented. Firstly, the description of the two-sided matching problem with uncertain scores is given. Secondly, the satisfaction degrees of each agent toward the agents on the other side are calculated. A multi-objective optimization model to maximize the satisfaction degrees of agents is set up. Considering the important degree of each agent in each side, the multi-objective optimization model is transformed into a bi-objective optimization model. Furthermore, the bi-objective ptimization model transformed into a single-objective model by using the linear weighted method. The matching alternative can be determined by solving the single-objective model.Lastly, an example is given to illustrate the validity of the proposed method.
\end{abstract}

Keywords: two-sided matching; uncertain score; satisfaction degree; optimization model

\section{Introduction}

The two-sided matching problems widely exist in the real world. Examples contain the stable marriage problem [17], the CEOs selection problem [5-8], the college admission problem [9-12], the employee selection problem [13-15], and the personnel assignment problem [16-18]. Since the reasonable and effective matching alternative has a promoting effect for improving the efficiency of economic management activities and the satisfaction degrees of two-sided agents, studying on the two-sided matching problems has important theoretical significance and practical application value.

Gale and Shapley study initially the famous marriage and college admission model [19]. A fundamental concept in the two-sided matching markets is that of stability. A two-sided matching is stable if there is no pair of one agent and another agent on the other side who likes each other better than their current partners. Then Roth [20] presents explicitly the concept of two-sided market, and shows that a number of models in labor markets 1ave surprising implications about the common and conflicting interests of the agents and about the incentives they face. Following that, various methods, techniques and algorithms have been proposed for solving the two-sided matching problem from different point of view. For example, Boon and Sierksma match positions with players in soccer team formation using the linear optimization models, and apply this model to American football, ice and field hockey, and many other management or project teams [21]. Manlove, et al., give a 2-approximation algorithm for the stable marriage problem with uncertain lists and ties [22]. Ehlers studies the truncation strategies in matching markets using the deferred acceptance algorithm [23]. Kim and Camera study a decentralized trading model, where a finite number of heterogeneous capacity-constrained sellers compete for a finite number of homogeneous buyers, by posting prices [24]. This study makes two contributions: a 
substantive contribution is to establish uniqueness of symmetric equilibrium; a methodological contribution is to develop a tool based on directional derivatives to characterize equilibrium.

Prior studies have made significant contributions to solving the two-sided matching problems from the perspective of theory, method and application. However, the two-sided matching problem with scores has received very little attention. On the one hand, in some practical problem, the preferences provided by agents may be uncertain scores. But the existing studies seldom consider solving this kind of problem. On the other hand, for each agent, it needs to consider the satisfaction degree of each agent (the relationship between the score and the satisfaction degree is usually not linear). But most of the existing studies don't concern about satisfaction degrees. Therefore, how to consider satisfaction degrees of agents in the two-sided matching problem with uncertain scores is a valuable research topic. This is the motivation of this study.

The objective of this paper is to propose a method for solving the two-sided matching problem with uncertain scores. Firstly, uncertain score matrixes are transformed into satisfaction degree matrixes. Then, a multi-objective optimization model is built, where the considered sub-objectives are to maximize satisfaction degree of each agent. Further, by using the linear weighted method twice, the multi-objective optimization model is solved. Based on the solution, the matching alternative can be obtained.

The remainder of this paper is arranged as follows. Section 2 formulates the two-sided matching problem with uncertain scores. Section 3 proposes amethod to solve the two-sided matching problem with uncertain scores. Section 4 presents a numerical example to show the use of the proposed method. Section 5 summarizes the nam features of the proposed method.

\section{Description of the Problemp}

This paper considers the two-sided matching problem with uncertain scores. Let $P=\left\{P_{1}, P_{2}, \ldots, P_{m}\right\} \quad(m \geq 2)$ be the set of agents of side $P$, where $P_{i}$ denotes the $i$ th agent of side $P$; Let $Q=\left\{Q_{1}, Q_{2}, \ldots, Q_{n}\right\}(m \leq n)$ be the set of agents of side $Q$, where $Q_{j}$ denotes the $j$ th agent of side $Q$. Let $I=\{1,2, \ldots, m\}, J=\{1,2, \ldots, n\}$. Let $S=\left\{s_{1}, s_{2}, \ldots, s_{i}\right\}$ be the set of scores. Let $U_{P}=\left[\hat{u}_{i j}^{P}\right]_{m \times n}$ be the uncertain score matrix from side $R$ to $Q$, where $\bar{u}_{i j}^{P}$ denotes the uncertain score of agent $P_{i}$ over $Q_{j}$, $\widehat{u}_{i j}^{P}=\left[u_{i j}^{P L}, u_{i j}^{P R}\right], u_{i j}^{P L}, u_{i j}^{P R} \in S, u_{i j}^{P L} \leq u_{i j}^{P R}$. Let $U_{Q}=\left[\widehat{u}_{i j}^{Q}\right]_{m \times n}$ be the uncertain score matrix from side $Q$ to $P$, where $\widehat{u}_{i j}^{Q}$ denotes the uncertain score of agent $Q_{j}$ over $P_{i}$, $\widehat{u}_{i j}^{Q}=\left[u_{i j}^{Q L}, u_{i j}^{Q R}\right], u_{i j}^{Q L}, u_{i j}^{Q R} \in S, u_{i j}^{Q L} \leq u_{i j}^{Q R}$.

Remark 1. In different practical problems, the score set $S=\left\{s_{1}, s_{2}, \ldots, s_{l}\right\}$ may have different expressions. For example, $S=\left\{s_{1}=1\right.$ (complete unsatisfied), $s_{2}=3$ (very unsatisfied), $s_{3}=5$ (moderate), $s_{4}=7$ (very satisfied), $s_{5}=9$ (complete satisfied) $\}$, and $\mathrm{S}=\left\{\mathrm{s}_{1}=1\right.$ (absolute poor), $\mathrm{s}_{2}=2$ (very poor), $\mathrm{s}_{3}=4$ (poor), $\mathrm{s}_{4}=5$ (moderate), $\mathrm{s}_{5}=6$ (good), $\mathrm{s}_{6}=8$ (very good), $\mathrm{s}_{7}=9$ (complete good) $\}$, etc.

Remark 2. In the two-sided matching problem, if $\widehat{u}=\left[u^{L}, u^{R}\right]$ is an uncertain score of an agent towards another, then we regard that scores $u^{L}, u^{L+1}, \ldots$, and $u^{R}$ possess the same possibility. Motivated by this idea, we give the following definition.

Definition 1. Let $\widehat{u}=\left[u^{L}, u^{R}\right]$ be the uncertain score of an agent towards another, then the probability vector on $\hat{u}$ is represented by $\hat{p}_{\hat{u}}=\left(p_{\hat{u}}^{1}, \ldots, p_{\hat{u}}^{k}, \ldots, p_{\hat{u}}^{m}\right)$, where 


$$
p_{u}^{k}=\left\{\begin{array}{lc}
1 /(R-L+1), & L \leq k \leq R \\
0, & \text { otherwise }
\end{array}\right.
$$

Here $p_{\bar{u}}^{k}$ denotes the probability that score preference is $u^{k}$.

Definition 2. Let $\widehat{u}=\left[u^{L}, u^{R}\right]$ be the uncertain score of an agent towards another, and $\hat{p}_{\bar{u}}$ be the probability vector on $\hat{u}$, then the expectation of $\hat{u}$ is represented by $E(\hat{u})$, where

$$
E(\widehat{u})=\widehat{u} \otimes \widehat{p}_{\widehat{u}}=\sum_{k=L}^{R}\left(k \times p_{\widehat{u}}^{k}\right)
$$

According to reference [23-25], the notation description of the two-sided matching is given as follows.

Definition 3. A two-sided matching is a one-to-one mapping $\mu: P \cup Q \rightarrow P \cup Q$ such that (i) $\mu\left(P_{i}\right) \in Q$, (ii) $\mu\left(Q_{j}\right) \in P \bigcup\left\{Q_{j}\right\}$, (iii) $\mu\left(P_{i}\right)=Q_{k}$ iff $\mu\left(Q_{k}\right)=P_{i}$.

Remark 3. In Definition 3, $\mu\left(P_{i}\right)=Q_{k}$ denotes that $P_{\text {ind }} Q_{k}$ are matched with each other, $\mu\left(Q_{j}\right)=Q_{j}$ denotes that $Q_{j}$ is not matched. If $\mu(P)=Q_{k}$, then $\left(P, Q_{k}\right)$ is called a matching pair. For convenience, we note $\left(Q_{j}, Q\right)$ as the matching pair in the case of $\mu\left(Q_{j}\right)=Q_{j}$. Hence, a matching alternative can be represented by the set of n matching pairs, such as $\mu=\left\{\left(P_{1}, Q_{2}\right),\left(P_{2}, Q_{4}\right), \ldots,\left(Q_{3}, Q_{3}\right)\right\}$.

In sum, the problem concerned in this paper is how to obtain the reasonable matching alternative based on uncertain score matrixes $U_{P}=\left[\vec{u}^{l}\right]_{m \times n}$ and $U_{Q}=\left[\widehat{u}_{i j}^{Q}\right]_{m \times n}$.

\section{The Proposed Method}

\subsection{Satisfaction Degree}

In the considered two-sided matching problem, without loss of generality, the greater the score is, the greater the satisfaction degree is. If the score given by agent $P_{i}$ towards $Q_{j_{1}}$ is the greatest, $i, e, \mu_{i_{\mathrm{I}}}=\left[s_{l}, s_{l}\right]$, then the satisfaction degree of agent $P_{i}$ over $Q_{j_{1}}$ is the highest; if the score given by agent $P_{i}$ towards $Q_{j_{2}}$ is the lowest, i.e., $\hat{u}_{i j_{2}}^{P}=\left[s_{1}, s_{1}\right]$, then the satisfaction degree of gent $P_{i}$ over $Q_{j_{2}}$ is the lowest. For convenience, the satisfaction degrees are in interval $[0,1]$.

Definition 4. Let $d_{i j}^{P}$ be the satisfaction degree of agent $P_{i}$ over $Q_{j}$, and $d_{i j}^{Q}$ be the satisfactiondegree of agent $Q_{j}$ over $P_{i}$, then $d_{i j}^{P}$ and $d_{i j}^{Q}$ are given by

$$
\begin{array}{r}
d_{i j}^{P}=\left(\frac{E\left(\hat{u}_{i j}^{P}\right)}{s_{l}}\right)^{2}, i \in I, j \in J \\
d_{i j}^{Q}=\left(\frac{E\left(\hat{u}_{i j}^{Q}\right)}{s_{l}}\right)^{2}, i \in I, j \in J
\end{array}
$$

By Eqs. (1)-(3), uncertain score matrixes $U_{P}=\left[\widehat{u}_{i j}^{P}\right]_{m \times n}$ and $U_{Q}=\left[\widehat{u}_{i j}^{Q}\right]_{m \times n}$ can be transformed into satisfaction degree matrixes $D_{P}=\left[d_{i j}^{P}\right]_{m \times n}$ and $D_{Q}=\left[d_{i j}^{Q}\right]_{m \times n}$. 


\subsection{Model}

Based on satisfaction degree matrixes $D_{P}=\left[d_{i j}^{P}\right]_{m \times n}$ and $D_{Q}=\left[d_{i j}^{Q}\right]_{m \times n}$, we consider constructing an optimization model for solving the two-sided matching problem with uncertain scores.

Let $x_{i j}$ be an 0-1 variable, where $x_{i j}=0$ denotes $\mu\left(P_{i}\right) \neq Q_{j}, x_{i j}=1$ denotes $\mu\left(P_{i}\right)=Q_{j}$, then $x_{i j}=\left\{\begin{array}{ll}1, & \mu\left(P_{i}\right)=Q_{j} \\ 0, & \mu\left(P_{i}\right) \neq Q_{j}\end{array}\right.$. To maximize the satisfaction degree of each agent, the following multi-objective optimization model (4) can be set up:

$$
\begin{array}{ll}
\max & Z_{P_{i}}=\sum_{j=1}^{n} d_{i j}^{P} x_{i j}, i \in I \\
\max & Z_{Q_{j}}=\sum_{i=1}^{m} d_{i j}^{Q} x_{i j}, j \in J \\
\text { s.t. } & \sum_{j=1}^{n} x_{i j}=1, i \in I \\
& \sum_{i=1}^{m} x_{i j} \leq 1, j \in \mathbb{U}
\end{array}
$$

In model (4), Eqs. (4a) and (4b) are objective functions. the meaning of Eq. (4c) is that agent $P_{i}$ must match only an agent of side $Q$; the meaning of Eq. (4d) is that agent $Q_{j}$ matches at most an agent of side $P$

\subsection{Solution}

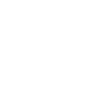

Let $w_{P_{i}}\left(0<w_{P_{i}}<1\right)$ be the important degree of agent $P_{i}$ in set $P$ such that $\sum_{i=1}^{m} w_{P_{i}}=1$; Let $w_{Q_{j}}\left(0<w_{\omega_{Q_{j}}}<1\right)$ be the important degree of agent $Q_{j}$ in set $Q$ such that $\sum_{j=1}^{n} w_{\omega_{Q_{j}}}=1$. Here, $w_{P_{i}}$ and $w_{Q_{j}}$ are usually given by the intermediary. If the statuses of agents in each side are equal, then $w_{P_{i}}=\frac{1}{m} w_{0}=\frac{1}{n}$. Furthermore, the linear weighted method is used to aggregate Eqs. (4a) and (4b). Then, model (4) can be transformed into the bi-objective optimization model (5):

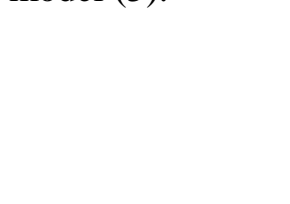

$$
\begin{array}{ll}
\max & Z_{P}=\sum_{i=1}^{m} w_{P_{i}} \sum_{j=1}^{n} d_{i j}^{P} x_{i j} \\
\max & Z_{Q}=\sum_{j=1}^{n} w_{Q_{j}} \sum_{i=1}^{m} d_{i j}^{Q} x_{i j} \\
\text { s.t. } & \sum_{j=1}^{n} x_{i j}=1, i \in I \\
& \sum_{i=1}^{m} x_{i j} \leq 1, j \in J \\
& x_{i j} \in\{0,1\}, i \in I, j \in J
\end{array}
$$


To solve model (5), the linear weighted method is also used. Let $w_{P}$ and $w_{Q}$ be the weight of objectives functions $Z_{P}$ and $Z_{Q}$, respectively, such that $0<w_{P}, w_{Q}<1, w_{P}+w_{Q}=1$, then model (5) is transformed into the single-objective optimization model (6):

$$
\begin{array}{ll}
\max & Z=\sum_{i=1}^{m} \sum_{j=1}^{n} \gamma_{i j} x_{i j} \\
\text { s.t. } & \sum_{j=1}^{n} x_{i j}=1, i \in I \\
& \sum_{i=1}^{m} x_{i j} \leq 1, j \in J \\
& x_{i j} \in\{0,1\}, i \in I, j \in J
\end{array}
$$

where $\gamma_{i j}=w_{P} w_{P_{i}} d_{i j}^{P}+w_{Q} w_{Q_{j}} d_{i j}^{Q}$. In the matching process, if the statuses of agents $P_{i}$ and $Q_{j}$ are the same, then we have $w_{P}=w_{Q}$, otherwise $w_{P} \neq w_{Q}$.

Since model (6) is a simple assignment model, the polynomial algorithm exists. Further, if the scale of problem is great, then we can develop speciar polynomial algorithm to solve. According to the multiple-objective programming theory, the optimal solution to model (6) is the efficient solution to model (5). Then the matching result is determined based on the obtained optimal solution.

\subsection{Procedure}

In sum, an algorithm is developed for solving the two-sided matching problem under uncertain score environment and its steps are provided as follows:

Step 1. Transform uncertain score matrixes $U_{P}=\left[\widehat{u}_{i j}^{P}\right]_{m \times n}$ and $U_{Q}=\left[\widehat{u}_{i j}^{Q}\right]_{m \times n}$ into satisfaction degree matrixes $D_{p}=\left[d_{i j}^{P}\right]_{m \times n}$ and $D_{Q}=\left[d_{i j}^{Q}\right]_{m \times n}$ by Eqs. (1)-(3).

Step 2. Built the multiple-objective optimization model (4) based on satisfaction degree matrices $D_{P}=\left[d_{i, j}^{P} \cap_{m \times n}\right.$ and $D_{Q}=\left[d^{Q}\right]_{m \times n}$.

Step 3. Transform model (4) into model (5) and then into model (6) by using the linear weighted method twice

Step 4. Obtain the matching alternative by solving model (6).

\section{Example|}

Huawe Technologies Co. Ltd. concentrates on the development of the lead-in correspondence equipments and produces, sell, serves. Huawei Technologies Co. Ltd. plans to bire staffs in five positions $\left(P_{1}, P_{2}, \ldots, P_{5}\right)$. After preliminary screening, eight applicants $\left(Q_{1}, Q_{2}, \ldots, Q_{8}\right)$ enter into the final assessment. Each position is held by an applicant, and each applicant is assigned to only a position. The experts from the position departments evaluate the applicants from four perspectives: personality, creative ability, previous experience, and human relationship skill. The applicants evaluate the positions from three perspectives: salary and welfare, development space, and work environment. Suppose the score set $\mathrm{S}=\left\{\mathrm{s}_{1}=1\right.$ (complete unsatisfied), $\mathrm{s}_{2}=2$ (very unsatisfied), $\mathrm{s}_{3}=3$ (unsatisfied), $\mathrm{s}_{4}=4$ (moderate), 
$\mathrm{s}_{5}=5$ (satisfied), $\mathrm{s}_{6}=6$ (very satisfied), $\mathrm{s}_{7}=7$ (complete satisfied) $\}$. The uncertain score matrixes $U_{P}=\left[\widehat{u}_{i j}^{P}\right]_{5 \times 8}$ and $U_{Q}=\left[\widehat{u}_{i j}^{Q}\right]_{5 \times 8}$ are shown as follows.

$$
\begin{aligned}
& U_{P}=\left[\begin{array}{llllllll}
{\left[s_{2}, s_{4}\right]} & {\left[s_{3}, s_{4}\right]} & {\left[s_{1}, s_{3}\right]} & {\left[s_{6}, s_{6}\right]} & {\left[s_{2}, s_{4}\right]} & {\left[s_{3}, s_{5}\right]} & {\left[s_{2}, s_{3}\right]} & {\left[s_{6}, s_{7}\right]} \\
{\left[s_{3}, s_{4}\right]} & {\left[s_{2}, s_{2}\right]} & {\left[s_{3}, s_{3}\right]} & {\left[s_{3}, s_{4}\right]} & {\left[s_{5}, s_{5}\right]} & {\left[s_{3}, s_{3}\right]} & {\left[s_{6}, s_{6}\right]} & {\left[s_{3}, s_{4}\right]} \\
{\left[s_{1}, s_{3}\right]} & {\left[s_{6}, s_{7}\right]} & {\left[s_{4}, s_{5}\right]} & {\left[s_{5}, s_{6}\right]} & {\left[s_{2}, s_{4}\right]} & {\left[s_{2}, s_{2}\right]} & {\left[s_{3}, s_{5}\right]} & {\left[s_{5}, s_{6}\right]} \\
{\left[s_{4}, s_{4}\right]} & {\left[s_{3}, s_{5}\right]} & {\left[s_{3}, s_{3}\right]} & {\left[s_{4}, s_{5}\right]} & {\left[s_{4}, s_{4}\right]} & {\left[s_{3}, s_{4}\right]} & {\left[s_{2}, s_{3}\right]} & {\left[s_{1}, s_{2}\right]} \\
{\left[s_{5}, s_{6}\right]} & {\left[s_{2}, s_{4}\right]} & {\left[s_{4}, s_{6}\right]} & {\left[s_{6}, s_{7}\right]} & {\left[s_{3}, s_{3}\right]} & {\left[s_{3}, s_{5}\right]} & {\left[s_{4}, s_{5}\right]} & {\left[s_{2}, s_{4}\right]}
\end{array}\right] \\
& U_{Q}=\left[\begin{array}{llllllll}
{\left[s_{3}, s_{5}\right]} & {\left[s_{2}, s_{3}\right]} & {\left[s_{6}, s_{7}\right]} & {\left[s_{3}, s_{4}\right]} & {\left[s_{2}, s_{3}\right]} & {\left[s_{6}, s_{6}\right]} & {\left[s_{3}, s_{4}\right]} & {\left[s_{3}, s_{5}\right]} \\
{\left[s_{3}, s_{4}\right]} & {\left[s_{5}, s_{5}\right]} & {\left[s_{3}, s_{3}\right]} & {\left[s_{5}, s_{6}\right]} & {\left[s_{3}, s_{3}\right]} & {\left[s_{3}, s_{4}\right]} & {\left[s_{2}, s_{2}\right]} & {\left[s_{6}, s_{6}\right]} \\
{\left[s_{5}, s_{6}\right]} & {\left[s_{2}, s_{4}\right]} & {\left[s_{2}, s_{2}\right]} & {\left[s_{4}, s_{5}\right]} & {\left[s_{2}, s_{4}\right]} & {\left[s_{3}, s_{4}\right]} & {\left[s_{1}, s_{3}\right]} & {\left[s_{2}, s_{4}\right.} \\
{\left[s_{4}, s_{5}\right]} & {\left[s_{4}, s_{4}\right]} & {\left[s_{3}, s_{4}\right]} & {\left[s_{5}, s_{6}\right]} & {\left[s_{3}, s_{4}\right]} & {\left[s_{2} s_{2}\right]} & {\left[s_{3}, s_{3}\right]} & {\left[s_{3}, s\right]} \\
{\left[s_{6}, s_{7}\right]} & {\left[s_{3}, s_{3}\right]} & {\left[s_{5}, s_{5}\right]} & {\left[s_{2}, s_{4}\right]} & {\left[s_{5}, s_{6}\right]} & \left.s_{6}, s_{3}\right] & {\left[s_{4}, s_{5}\right.} & {\left[s_{2}, s_{3}\right]}
\end{array}\right] \text {. }
\end{aligned}
$$

To obtain the matching alternative, a detailed description of the matching process is given below.

Step 1. According to uncertain score matrixes $U_{P}=\left[\hat{u}_{i{ }^{\prime}}^{P}\right]_{5,8}$ and $U_{Q}=\left[\hat{u}_{i j}^{Q}\right]_{5 \times 8}$, satisfaction degrees matrixes $D_{P}=\left[d_{i j}^{P}\right]_{5 \times 8}$ and $D_{Q}=\left[d^{Q} \Omega\right.$ are built by Eqs, (1)-(3), which are shown in Tables 1 and 2, respectively.

Table 1. Satisfaction Degrees Matrixes $D_{P}=\left[d_{i j}^{P}\right]_{5 \times 8}$

\begin{tabular}{ccccccccc}
\hline$d_{i j}^{P}$ & $Q_{1}$ & $Q_{2}$ & $Q_{3}$ & $Q_{4}$ & $Q_{5}$ & $Q_{6}$ & $Q_{7}$ & $Q_{8}$ \\
\hline$P_{1}$ & 0.1837 & 0.25 & 0.0816 & 0.7347 & 0.1837 & 0.3265 & 0.1276 & 0.8622 \\
$P_{2}$ & 0.25 & 0.0816 & 0.1837 & 0.25 & 0.5102 & 0.1837 & 0.7347 & 0.25 \\
$P_{3}$ & 0.0816 & 0.8622 & 0.4133 & 0.6173 & 0.1837 & 0.0816 & 0.3265 & 0.6173 \\
$P_{4}$ & 0.3265 & 0.3265 & 0.1837 & 0.4133 & 0.3265 & 0.25 & 0.1276 & 0.0459 \\
$P_{5}$ & 0.6173 & 0.1837 & 0.5102 & 0.8622 & 0.1837 & 0.5102 & 0.4133 & 0.1837 \\
\hline
\end{tabular}

Table 2. Satisfaction Degrees Matrixes $D_{Q}=\left[d_{i j}^{Q}\right]_{5 \times 8}$

\begin{tabular}{ccccccccc}
\hline$d_{i j}^{Q}$ & $Q_{1}$ & $Q_{2}$ & $Q_{3}$ & $Q_{4}$ & $Q_{5}$ & $Q_{6}$ & $Q_{7}$ & $Q_{8}$ \\
\hline$P_{1}$ & 0.3265 & 0.1276 & 0.8622 & 0.25 & 0.1276 & 0.7347 & 0.25 & 0.3265 \\
$P_{2}$ & 0.25 & 0.5102 & 0.1837 & 0.6173 & 0.1837 & 0.25 & 0.0816 & 0.7347 \\
$P_{3}$ & 0.6173 & 0.1837 & 0.0816 & 0.4133 & 0.1837 & 0.25 & 0.0816 & 0.1837 \\
$P_{4}$ & 0.4133 & 0.3265 & 0.25 & 0.6173 & 0.25 & 0.0816 & 0.1837 & 0.25 \\
$P_{5}$ & 0.8622 & 0.1837 & 0.5102 & 0.1837 & 0.6173 & 0.8622 & 0.4133 & 0.1276 \\
\hline
\end{tabular}

Step 2. Based on satisfaction degree matrices $D_{P}=\left[d_{i j}^{P}\right]_{5 \times 8}$ and $D_{Q}=\left[d_{i j}^{Q}\right]_{5 \times 8}$, model (4) is set up, i.e., 


$$
\begin{array}{ll}
\max & Z_{P_{i}}=\sum_{j=1}^{8} d_{i j}^{P} x_{i j}, i \in I \\
\max & Z_{Q_{j}}=\sum_{i=1}^{5} d_{i j}^{Q} x_{i j}, j \in J \\
\text { s.t. } & \sum_{j=1}^{8} x_{i j}=1, i \in I \\
& \sum_{i=1}^{5} x_{i j} \leq 1, i \in I \\
& x_{i j} \in\{0,1\}, i \in I, j \in J
\end{array}
$$

where $I=\{1,2, \ldots, 5\}, J=\{1,2, \ldots, 8\}$.

$$
\text { Step 3: Assume } w_{P_{i}}=\left\{\begin{array}{l}
\frac{1}{4}, i=1,2, \\
\frac{1}{6}, i=3,4,5,
\end{array} w_{Q_{j}}=\left\{\begin{array}{l}
\frac{1}{6}, j=1,2,3, \\
\frac{1}{10}, j=4, \ldots
\end{array}\right\}\right. \text {, then by using the linear }
$$
weighted method, model (4) is transformed into model (5), i.e.,

$$
\begin{gathered}
\max Z_{P}=\sum_{i=1}^{5} w_{p} \sum_{j=1}^{8} d_{i j}^{P} x_{i j} \\
\max Z_{j=1}^{8} w_{Q_{j}} \sum_{i=1}^{5} d_{i j}^{O} x_{i j} \\
\sum_{i j}^{5} \in\{0,1\}, i \in I, j \in J
\end{gathered}
$$

Furthermore, suppose $w_{P}=\frac{3}{5}$ and $w_{Q}=\frac{2}{5}$, then by using the linear weighted method, model (5) is transformed into model (6), i.e.,

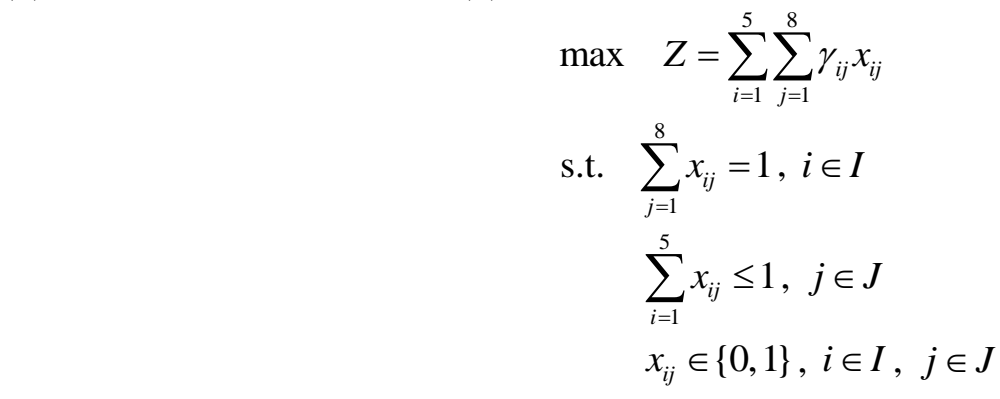

where coefficient matrix $\Gamma=\left[\gamma_{i j}\right]_{5 \times 8}$ is shown in Table 3 . 
Table 3. Coefficient Matrix $\Gamma=\left[\gamma_{i j}\right]_{5 \times 8}$

\begin{tabular}{ccccccccc}
\hline$\gamma_{i j}$ & $Q_{1}$ & $Q_{2}$ & $Q_{3}$ & $Q_{4}$ & $Q_{5}$ & $Q_{6}$ & $Q_{7}$ & $Q_{8}$ \\
\hline$P_{1}$ & 0.0493 & 0.046 & 0.0697 & 0.1202 & 0.0327 & 0.0784 & 0.0291 & 0.1424 \\
$P_{2}$ & 0.0542 & 0.0463 & 0.0398 & 0.0622 & 0.0839 & 0.0376 & 0.1135 & 0.0669 \\
$P_{3}$ & 0.0493 & 0.0985 & 0.0468 & 0.0783 & 0.0257 & 0.0182 & 0.0359 & 0.0691 \\
$P_{4}$ & 0.0602 & 0.0544 & 0.035 & 0.066 & 0.0427 & 0.0283 & 0.0201 & 0.0146 \\
$P_{5}$ & 0.1192 & 0.0306 & 0.085 & 0.0936 & 0.0431 & 0.0855 & 0.0579 & 0.0235 \\
\hline
\end{tabular}

Step 4: By solving model (6), the unique optimal solution is obtained, i.e.,

$$
X^{*}=\left[x_{i j}^{*}\right]_{5 \times 8}=\left[\begin{array}{cccccccc}
0 & 0 & 0 & 0 & 0 & 0 & 0 & 1 \\
0 & 0 & 0 & 0 & 0 & 0 & 1 & 0 \\
0 & 1 & 0 & 0 & 0 & 0 & 0 & 0 \\
0 & 0 & 0 & 1 & 0 & 0 & 0 & 0 \\
1 & 0 & 0 & 0 & 0 & 0 & 0 & 0
\end{array}\right]
$$

According to the unique optimal solution $X^{*}$ the matching alternative $\mu^{*}$ can be

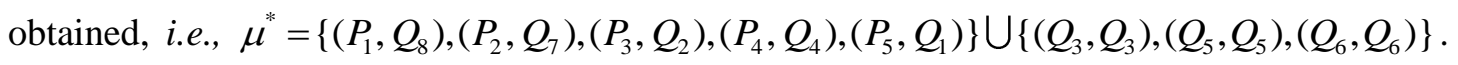
In other words, position $P_{1}$ matches with applicant $Q_{8}$, position $P_{2}$ matches with applicant $Q_{7}$, position $P_{3}$ matches with applicant $Q_{2}$ position $P_{4}$ matches with applicant $Q_{4}$, position $P_{5}$ matches with applicant $Q_{1}$, applicants $Q_{3}, Q_{5}$ and $Q_{6}$ are unmatched.

\section{Conclusion}

This paper presents a new method for solving the two-sided matching problem with uncertain scores. The formulas of the satisfaction degree are firstly given. Then, a multiobjective optimization model considering the matching constraints is set up. The objective of the model is to maxinize the satisfaction degree of each agent. By solving the model, the matching alternative is obtained, The proposed method has two characteristics as follows.

First, in the proposed method, the satisfaction degrees of agents are considered. This is usually absent in the existing methods. Second, the proposed method is theoretically sound and computationally simple, which provides a new way to solve the two-sided matching problem with uncertain scores and can be adopted for practical use.

\section{Acknowledgment}

This work was partly supported by the National Natural Science Foundation of China (Project Nos. 71261007, 71261006, 71361021), the Humanities and Social Science Foundation of the Ministry of Education of China (Project No. 12YJC630080), Natural Science Fund of Jiangxi Province (Project No. 20132BAB201015), and Science and Technology Research Project of the Department of Education of Jiangxi Province (Project No. GJJ13292).

\section{References}

[1] C. C. Huang and T. Kavitha, Inform. Comput., vol. 222, no. 180, (2013).

[2] F. Tamás, R. W. Irving and D. F. Manlove, Theor. Compu. Sci. vol. 381, no. 162, (2007).

[3] S. Lauermann, and G. Nöldeke, J. Econ. Theory, vol. 151, no. 163, (2014). 
[4] D. Nizamogullari and İ. Özkal-Sanver, Math. Social Sciences, vol. 69, no. 34, (2014).

[5] X. Gabaix, and A. Landier, Q. J. Econ., vol. 123, no. 49, (2008).

[6] S. Allgood, K. A. Farrell and R. Kamal, Journal of Corporate Finance, vol. 18, no. 1051, (2012).

[7] L. A. Taylor, J. Financ. Econo., vol. 108, no. 79, (2013).

[8] F. Gallego and B. Larrain, J. Comp. Econ., vol. 40, no. 621, (2012).

[9] J. Alcalde and A. Romero-Medina, Econ. Lett., vol. 86, no. 153, (2005).

[10] W. C. Chen and Y. C. Kao, Econ. Lett., vol. 122, no. 296, (2014).

[11] M. Koljatic, M. Silva and R. Cofré, Int. J. Educ. Dev., vol. 33, no. 106, (2013).

[12] M. Koljatic, M. Silva and C. Rodrigo, Int. J. Educ. Dev., vol. 33, no. 106, (2013).

[13] A. Golec and E. Kahya, Comput. Ind. Eng., vol. 52, no. 143, (2007).

[14] R. Mendes, G. J. V. D. Berg and M. Lindeboom, Labour Economics, vol. 17, no. 919, (2010).

[15] G. A. Wang, H. Atabakhsh and H. Chen, Decision Support Systems, vol. 51, no. 413, (2011).

[16] I. Korkmaz, H. Gökçen and T. Çetinyokuş, Inform. Sciences, vol. 178, no. 2915, (2008).

[17] K. Burdett, abd M. Coles, Q. J. Econ., vol. 112, no. 141, (1997).

[18] S. Y. Lin, S. J. Horng, T. W. Kao, et al., Applied Soft Computing, vol. 12, no. 2840, (2012).

[19] D. Gale and L. Shapley, Am. Math. Mon., vol. 69, no. 9, (1962).

[20] A. E. Roth, Eur. Econ. Rev., vol. 27, no. 75, (1985).

[21] B. H. Boon and G. Sierksma, Eur. J. Oper. Res., vol. 148, no. 277, (2003)

[22] D. F. Manlove, R. W. Irving, K. Iwama, S. Miyazaki, and Y. Moritab, Theor. Comput. Sci., vol. 276 , no. 261, (2002).

[23] L. Ehlers, Math. Oper. Res., vol. 33, no. 327, (2008).

[24] J. Kim and G. Camera, Journal of Economic Theory, vol. 151, no. 248, (2014).

[25] F. Echenique, Math. Oper. Res., vol. 33, no. 757, (2008)

\section{Author}

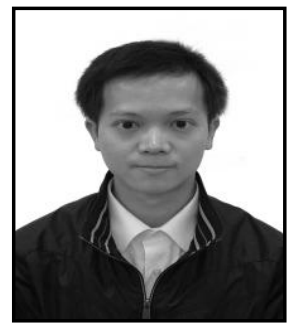

Qi Yue, he was born in 1983. He received the Ph.D. degree in school of business administration from northeastern university, shenyang, China, in 2011 . He is currenty a lecturer in the school of information management at jiangx nniversity of finance and economics. He is the author or coauthor of near forty papers published in international and local journals. His current interests include information science, management sejence, decision analysis and operations research. E-mail: yueqichina@126.com.

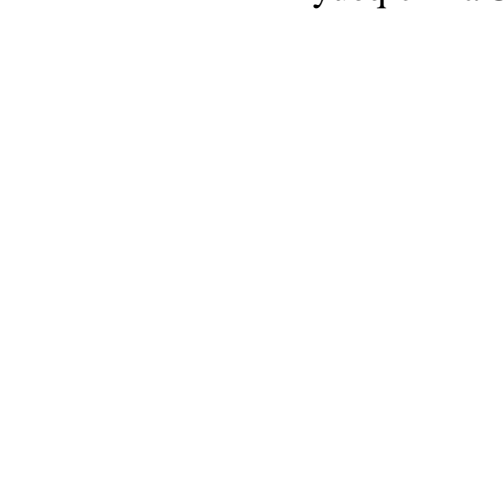


International Journal of Multimedia and Ubiquitous Engineering Vol. 9, No. 12 (2014)

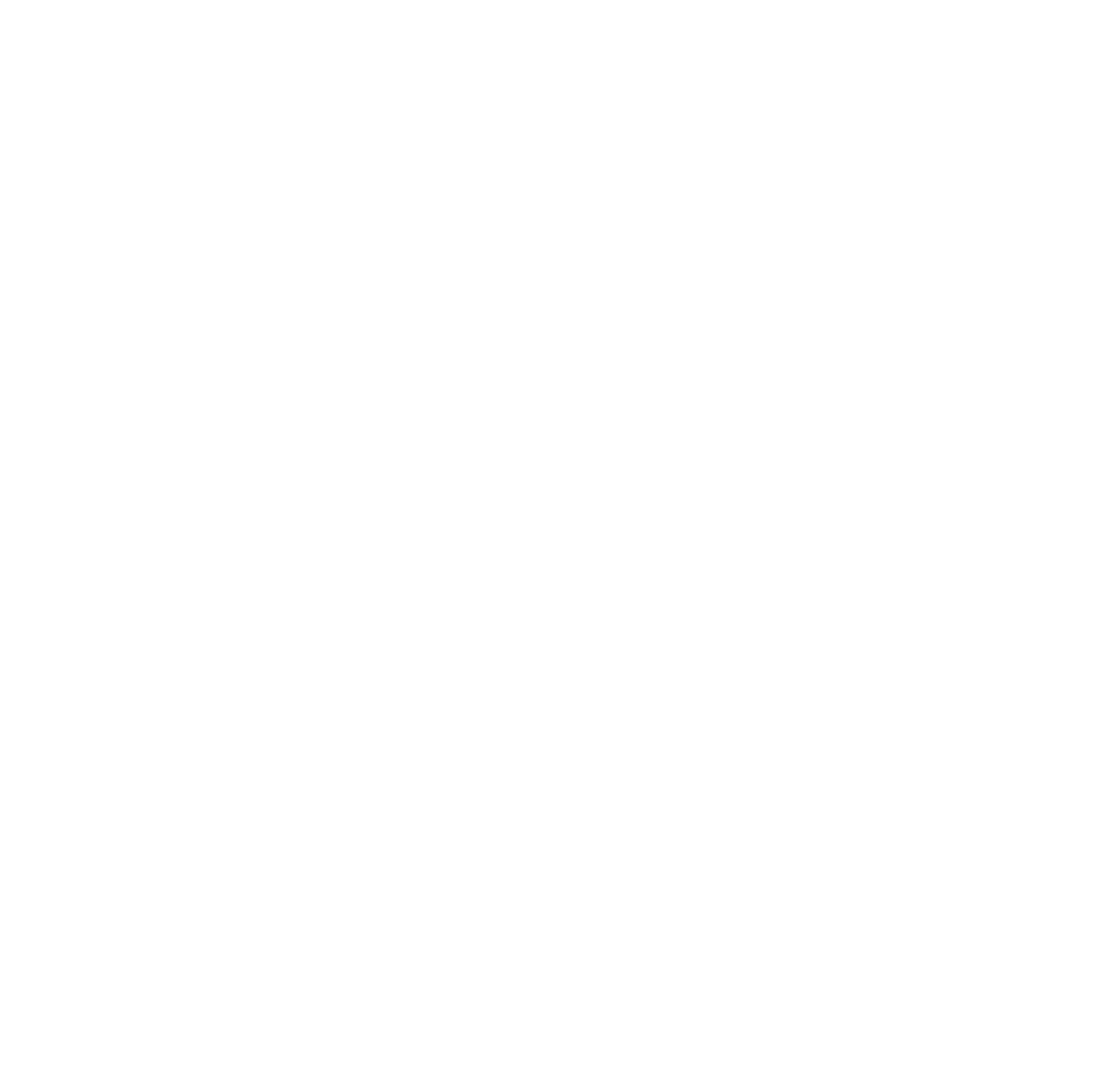

